\title{
A utilização de material didático manipulável e da geometria fractal para o aprendizado dos conceitos de área e perímetro de quadrado: um estudo de caso envolvendo uma estudante com baixa visão
}

The use of manipulative didactic material and fractal geometry for the learning of the concepts of area and square perimeter: a case study involving a student with low vision

La utilización de material didáctico manipulable y de la geometría fractal para el aprendizaje de los conceptos de área y perímetro de cuadrado: un estudio de caso involucrando a una estudiante con baja visión

\author{
* Ana Paula Poffo Koepsel \\ Mestre na Universidade Regional de Blumenau (FURB), Blumenau, Santa Catarina, Brasil. \\ annapaulapoffo@hotmail.com \\ ** Tânia Baier \\ Professora doutora na Universidade Regional de Blumenau (FURB), Blumenau, Santa Catarina, Brasil.
}

Recebido: 21 de junho de 2017

Aprovado: 7 de julho de 2017

\section{RESUMO}

Este artigo apresenta o relato de um estudo de caso relacionado com os conteúdos matemáticos área e perímetro de quadrados. Visando contemplar temas da Matemática criados na contemporaneidade, foi estudado o fractal Tapete de Sierpinski, cuja construção está relacionada com quadrados. A vivência pedagógica ocorreu com uma estudante que tem baixa visão, do $6^{\circ}$ ano do Ensino Fundamental de uma escola pública localizada no município de Blumenau (SC), com o intuito de contribuir para o aprendizado dos conceitos de área e perímetro de quadrados. No texto, inicialmente, são apresentadas algumas considerações para o atendimento educacional de pessoas com deficiência visual. Em seguida são abordados os tópicos de geometria euclidiana e fractal, que foram enfocados na ação pedagógica relatada. Finalizando, é descrito o material didático manipulável, cortado em E.V.A., previamente analisado e aprovado pela professora da sala multifuncional da escola frequentada pela estudante, sendo relatada a sua aplicação e apresentados os resultados obtidos. A estudante não apresentou dificuldades em identificar as figuras apresentadas no material, realizou de modo correto a contagem dos quadrados 
e compreendeu os conceitos de área e perímetro de quadrados. No entanto, encontrou dificuldade no uso da régua adaptada, confundindo a origem do objeto com o zero onde deve ser iniciada a medida. A manipulação do material didático descrito neste artigo auxiliou no entendimento dos conceitos área e perímetro de quadrados e também possibilitou a construção, por meio do encaixe de peças, do fractal Tapete de Sierpinski.

Palavras-chave: Baixa visão; Geometria fractal; Área; Perímetro.

\section{ABSTRACT}

This article presents the report of a case study related to the mathematical contents area and perimeter of squares. Aiming to contemplate Mathematical themes created in the contemporaneity, the fractal Sierpinski Carpet, whose construction is related to squares, was studied. The pedagogical experience occurred with a student who has low vision, from the 6th grade of Elementary School of a public school located in the city of Blumenau (SC), with the intention of contributing to the learning of the concepts of area and perimeter of squares. In the text, initially, some considerations are presented for the educational attendance of visually impaired people. Next, the topics of Euclidean and fractal geometry are addressed, which were focused on the pedagogical action reported. Finally, it is described the manipulative didactic material, cut in E.V.A., previously analyzed and approved by the teacher of the multifunctional room of the school attended by the student, being reported its application and presented the results obtained. The student did not present difficulties in identifying the figures presented in the material, realized correctly the counting of squares and understood the concepts of area and perimeter of squares. However, it found difficulty in using the adapted ruler, confusing the origin of the object with zero where the measure should be initiated. The manipulation of the didactic material described in this article helped in the understanding of the concepts of area and perimeter of squares and also made possible the construction, through the fitting of pieces, of the fractal Sierpinski Carpet.

Keywords: Low vision; Geometry; Area; Perimeter.

\section{RESUMEN}

Este artículo presenta el relato de un estudio de caso relacionado con los contenidos matemáticos área y perímetro de cuadrados. Con el objeto de contemplar temas de la Matemática creados en la contemporaneidad, se estudió el fractal alfabético de Sierpinski, cuya construcción está relacionada con cuadrados. La vivencia pedagógica ocurrió con una estudiante que tiene baja visión, del 6ํaño de la Enseñanza Fundamental de una escuela pública ubicada en el municipio de Blumenau (SC), con el objetivo de contribuir al aprendizaje de los conceptos de área y perímetro de cuadrados. En el texto, inicialmente, se presentan algunas consideraciones para la atención educativa de personas con 
discapacidad visual. A continuación se abordan los tópicos de geometría euclidiana y fractal, que se enfocaron en la acción pedagógica relatada. En el presente trabajo se analizan los resultados obtenidos en el estudio de los resultados obtenidos en el estudio. La estudiante no presentó dificultades en identificar las figuras presentadas en el material, realizó de modo correcto el recuento de los cuadrados y comprendió los conceptos de área y perímetro de cuadrados. Sin embargo, encontró dificultad en el uso de la regla adaptada, confundiendo el origen del objeto con el cero donde debe iniciarse la medida. La manipulación del material didáctico descrito en este artículo ayudó en el entendimiento de los conceptos área y perímetro de cuadrados y también posibilitó la construcción, por medio del encaje de piezas, del fractal Tapete de Sierpinski.

Palabras clave:

Baja visión; Geometría fractal; zona; Perímetro.

\section{Educação centrada no aluno}

Todas as pessoas possuem necessidades educacionais diferenciadas, que precisam ser consideradas pelo professor, principalmente quando estão presentes em sua sala de aula estudantes com alguma deficiência. Cada estudante tem seu próprio ritmo de aprendizagem, com habilidades diferenciadas, cada qual apresentando possibilidades nas mais diversas áreas do conhecimento e apreciando ao seu modo os temas abordados nos componentes curriculares. Bicudo (2006, p. 97) destaca: "O professor deve ser autêntico, imaginativo e providenciar os recursos importantes e diferenciados para a aprendizagem. Sua postura deve ser a de aceitação do aluno em suas possibilidades". Essa autora apresenta o seu entendimento sobre a educação centrada no aluno, sendo que um dos objetivos é a descoberta de si mesmo, que conduz ao entendimento de que cada estudante deve conhecer suas capacidades, compreendendo que ninguém é igual aos outros e cada qual tem possibilidades a serem desveladas. "Dentre os objetivos da educação centrada no aluno está a descoberta de si mesmo, ou seja, a proposta de levar o ser que aprende a se conhecer" (BICUDO, 2006, p. 86, grifo do autor).

Para essa autora, seguindo a fenomenologia como filosofia de educação, o professor se preocupa com as questões relacionadas com a inclusão escolar. Ao planejar atividades didáticas, assumindo a atitude fenomenológica, o professor prioriza o estudante, buscando compreender o seu modo de ser e objetivando o entendimento dos conteúdos curriculares, e "[...] não se trata de uma educação voltada apenas para o conhecimento do 
idiossincrático e individual. Procura, também, focar o conhecimento do igual e do diferente entre os seres humanos" (BICUDO, 2006, p. 87). Desse modo, a educação centrada no aluno não foca um conteúdo a ser ensinado, mas sim valoriza as diferenças e as possibilidades de cada estudante.

A seguir, privilegiando a educação centrada no aluno, enfocaremos o estudante com deficiência visual, objetivando o estudo de área e perímetro de quadrados incluindo noções elementares de geometria fractal. Inicialmente serão apresentadas definições de deficiência visual conforme documentos oficiais brasileiros e recomendações de pesquisadores que investigaram o atendimento aos estudantes com deficiência visual.

\section{Considerações para o atendimento educacional de pessoas com deficiência visual}

Considerando que a pessoa com deficiência visual é aquela que possui baixa visão ou cegueira, de acordo com a portaria n 3.128/08 do Ministério da Saúde temos que

[...] baixa visão ou visão subnormal, quando o valor da acuidade visual corrigida no melhor olho é menor do que 0,3 e maior ou igual a 0,05 ou seu campo visual é menor do que $20^{\circ}$ no melhor olho com a melhor correção óptica [...] e considera-se cegueira quando esses valores encontram-se abaixo de 0,05 ou o campo visual menor do que 10 . (BRASIL, 2008)

Como vivemos em um mundo onde a visão é um dos sentidos mais utilizados para a comunicação e a interação entre as pessoas, e na escola não é diferente, a deficiência visual dificulta a aprendizagem, principalmente dos temas do componente curricular Matemática. Os autores Shimazaki, Silva e Viginheski (2016) afirmam que os estudantes com deficiência visual possuem necessidades diferenciadas, precisando de adaptações dos textos didáticos e da utilização de distintos materiais para auxiliar na construção do conhecimento. Assim sendo, é necessário que os professores busquem novas metodologias de ensino que possam atender às necessidades educativas destes estudantes. Essas adaptações que o professor deve realizar para atender os estudantes com deficiência visual se caracterizam como recursos de tecnologia assistiva, definida do seguinte modo:

Tecnologia Assistiva é uma área do conhecimento, de característica interdisciplinar, que engloba produtos, recursos, metodologias, estratégias, práticas e serviços que objetivam promover a funcionalidade, relacionada à atividade e participação, de pessoas com deficiência, incapacidades ou 
mobilidade reduzida, visando sua autonomia, independência, qualidade de vida e inclusão social. (BRASIL, 2009, p.26)

O ensino de Matemática tradicional não é suficiente para atender às necessidades dos estudantes com deficiências, conforme ressaltam os autores Shimazaki, Silva e Viginheski (2016, p. 150):

O ensino de Matemática, fundamentado na prática pedagógica tradicional, que reduz o ensino da disciplina à apresentação de conceitos já elaborados, ao uso do livro didático, do quadro e do giz, pode ser considerado como uma prática pedagógica excludente, uma vez que não atende à diversidade, pois não proporciona aos estudantes, especialmente aos estudantes com deficiências, diferentes caminhos e possibilidades para a elaboração do conhecimento científico, conforme suas necessidades.

Ao deparar-se com um estudante com deficiência visual, surgem dúvidas no professor de como proceder porque ele necessita de métodos de ensino diferenciados. Sá, Campos e Silva (2007) explicam que, para estudantes com deficiência visual compreenderem algum tema, é necessário coletar as informações através dos sentidos remanescentes. Os mesmos autores apontam que a comunicação também é muito importante na sala de aula, recomendando que tanto professores como colegas de turma adquiram "[...] o hábito de evitar a comunicação gestual e visual na interação com esses alunos" (2007, p. 22).

Um recurso pedagógico a ser utilizado pelo professor, visando proporcionar a utilização dos sentidos remanescentes e possibilitar a aprendizagem de estudantes com deficiência visual, é o uso de materiais didáticos manipuláveis. Sá, Campos e Silva (2007) destacam que os professores devem utilizar em sala de aula outros materiais, não apenas aqueles de estímulos visuais, para estimular a exploração e o desenvolvimento dos sentidos remanescentes.

No presente artigo, os conteúdos curriculares abordados são área e perímetro de quadrados e, visando abordar temas da Matemática criados na contemporaneidade, foi estudado o fractal Tapete de Sierpinski, cuja construção está relacionada com quadrados. $\mathrm{Na}$ próxima seção, estão apresentadas algumas ideias elementares da geometria euclidiana e da geometria fractal, relacionadas com os conceitos área e perímetro. Tal suporte teórico foi elaborado objetivando a construção de material didático manipulável adequado para estudantes com deficiência visual. 


\section{Geometrias: euclidiana e fractal}

O conceito da forma geométrica quadrada envolve as ideias de ângulo e lado, sendo que duas semirretas com a mesma origem formam um ângulo, a origem é conhecida como vértice e as semirretas são denominadas lados do ângulo (DANTE, 2004). A atividade pedagógica descrita a seguir está relacionada com o conceito de áreas e perímetros de formas geométricas quadradas. "A área de uma região plana é igual ao número de unidades necessárias para cobrir essa região" (DANTE, 2002a, p. 268). A ideia de perímetro pode ser explicada como sendo o comprimento do contorno da forma geométrica, ou seja, perímetro "[...] é a soma das medidas dos comprimentos dos lados" (DANTE, 2002b, p. 226).

Benoit Mandelbrot, no livro Objectos Fractais, apresenta a sua definição dos termos objeto fractal ou fractal explicando que criou essas palavras "[...] a partir do adjectivo latino fractus, que significa «irregular» ou «quebrado»" (MANDELBROT, 1998, p. 13, grifo do autor). O mesmo autor considera o termo fractal original francês, "escrevendo-o como fractale [...] que depois se transmitiu ao inglês" (MANDELBROT, 1998, p. 171, grifo do autor).

Os fractais são utilizados para descrever formas irregulares que se encontram, por exemplo, nos modelos simplificados de litorais geográficos, cristais, flocos de neve, enfim, representações da irregularidade presente na Natureza. Eles são objetos matemáticos construídos sendo acrescentadas ou retiradas muitas partes indefinidamente. Os padrões característicos dos fractais são repetidamente observados em todas as escalas, ou seja, suas partes são, na forma, semelhantes ao todo. A geometria fractal "[...] é o estudo de diversos objetos, tanto matemáticos como naturais, que não são regulares, mas rugosos, porosos, ou fragmentados, sendo-o no mesmo grau em todas as escalas" (MANDELBROT, 1998, p. 207, grifo do autor).

Mandelbrot apresenta as seguintes características dos fractais:

a) As suas partes têm a mesma forma ou estrutura que o todo, estando porém a uma escala diferente e podendo estar um pouco deformadas. b) A sua forma é ou extremamente irregular ou extremamente interrompida ou fragmentada, assim como todo o resto, qualquer seja a escala de observação.

c) Contém «elementos distintos» cujas escalas são muito variadas e cobrem uma vasta gama. (MANDELBROT, 1998, p. 171, grifo do autor) 
Karas (2008) aponta que os fractais são figuras diferentes das estudadas na geometria euclidiana. São construídos a partir de processos iterativos, os quais, sendo teoricamente infinitos, impedem a sua representação fiel. Mandelbrot descreve "[...] a geometria fractal como sendo uma nova linguagem geométrica" (1998, p. 210, grifo do autor).

Alguns fractais estão relacionados com as formas geométricas estudadas no ensino fundamental: o Conjunto de Cantor e a Curva de Peano são construídos a partir de segmentos de reta; um triângulo equilátero fundamenta a construção da Ilha de Koch; o Tapete de Sierpinski se relaciona com o quadrado e será enfocado neste artigo.

De acordo com o Centro de Física Teórica e Computacional (2016, s/p) "[...] o tapete de Sierpinski é o conjunto resultante da remoção sucessiva do quadrado do centro, quando se divide um quadrado em nove quadrados". Para a construção do Tapete de Sierpinski, cujas primeiras iterações estão apresentadas na Figura 1, é aplicada a técnica de remoção, apresentada por Barbosa (2005, p. 44): “[...] partindo de um quadrado, dividindo-o em 9 pequenos quadrados congruentes, e eliminando o central. Em seguida, aplicar esse mesmo procedimento em cada um dos 8 quadrados restantes, e assim sucessivamente e iterativamente".

Figura 1 - Iterações iniciais do Tapete de Sierpinski
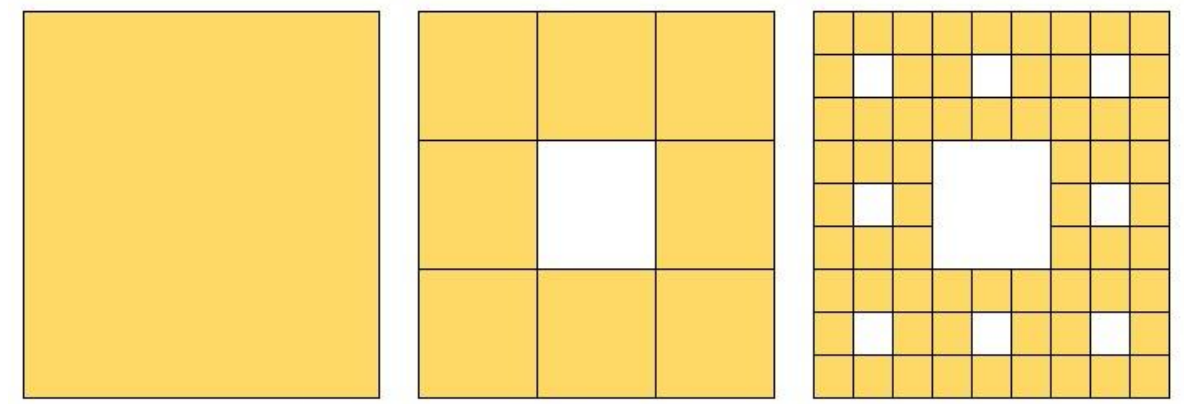

\section{Descrição do material didático}

O material didático foi elaborado a partir das orientações de Sá, Campos e Silva (2007) para adaptação de materiais a serem usados por estudantes com deficiência visual. Esses autores apontam que, para a elaboração de materiais didáticos para estudantes com deficiência visual, alguns critérios devem ser tomados para que haja eficácia na aplicação, como: a fidelidade da representação, o material deve ser o mais exato possível do original. 
O material deve ser agradável ao tato e, para ser usado por todos os estudantes da sala, ser envolvente para a visão. A adequação para a faixa etária e ao conteúdo é essencial. Outros critérios muito importantes são a dimensão e o tamanho, quando os objetos ou desenhos são muito pequenos não evidenciam detalhes de suas partes e podem ser facilmente perdidos, já quando o tamanho é exagerado afeta a apresentação do todo e dificulta a percepção global. O relevo também é importante porque deve ser facilmente percebido, e, se o material tiver várias partes, é necessário utilizar diferentes texturas para diferenciá-las. O material também deve resistir à exploração tátil, não provocar rejeição ao manuseio e não oferecer perigo aos estudantes (SÁ; CAMPOS; SILVA, 2007).

Os materiais didáticos elaborados foram previamente analisados e aprovados pela professora da sala multifuncional da escola que a estudante frequenta, porém, não estão disponíveis na secretaria informações detalhadas sobre o seu nível de deficiência visual. Observou-se que essa estudante efetua a leitura de números escritos em grandes dimensões com pincel marcador permanente preto sobre fundo branco, mas essa não é a estratégia pedagógica adotada sendo que todas as atividades didáticas por ela realizadas são previamente organizadas pelos professores das diferentes disciplinas e posteriormente traduzidas para o Braille. Para a elaboração do material didático descrito no presente artigo, foram utilizadas folhas de E.V.A, cola para E.V.A e bases para fixar as iterações. Para perceber as diferenças entre algumas formas euclidianas, foram recortados quadrados, triângulos, círculos e retângulos de tamanhos diferentes. Para a confecção das maquetes do Tapete de Sierpinski, foram seguidos os seguintes passos.

$1^{\circ}$ passo: De uma folha E.V.A foi cortado um quadrado de $18 \mathrm{~cm}$ de lado e colado em uma base. Após a secagem, foram cortadas com estilete 17 linhas na horizontal e 17 na vertical, para formar um quadriculado possível de ser tateado, com quadradinhos de lados medindo um centímetro.

$2^{\circ}$ passo: Foram cortados oito quadrados de $6 \mathrm{~cm}$ de lado e colados em uma base, representando a primeira iteração do Tapete de Sierpinski.

$3^{\circ}$ passo: No E.V.A foram recortados 64 quadrados de $2 \mathrm{~cm}$ de lado e colados na base para representar a segunda iteração do Tapete de Sierpinski.

Também foi construído o material usado para a avaliação do entendimento da construção do fractal Tapete de Sierpinski, sendo recortados em E.V.A nove quadrados de 
$8 \mathrm{~cm}$ de lado e quatro tiras de $1 \mathrm{~cm} \times 24 \mathrm{~cm}$ para a moldura da figura. As tiras foram coladas em uma base formando um quadrado de $24 \mathrm{~cm}$ de lado, de modo que, dentro dessa moldura possam ser encaixados os pequenos quadrados.

A figura 2 mostra as maquetes das primeiras iterações do fractal Tapete de Sierpinski e o material usado para a avaliação.

Figura 2 - Material didático Tapete de Sierpinsk

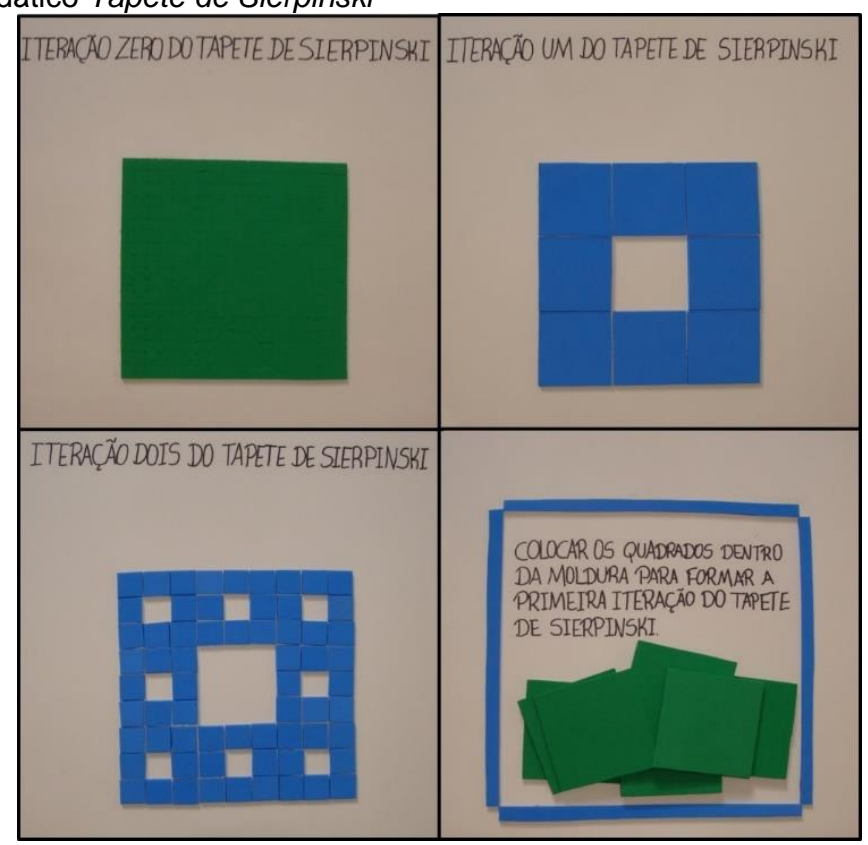

\section{Descrição da aplicação da atividade e resultados obtidos}

A aplicação da atividade ocorreu com uma estudante do $6^{\circ}$ ano do Ensino Fundamental de uma escola pública de Blumenau (SC) que tem baixa visão, durante duas aulas de 45 minutos. Juntamente com a sua turma, essa estudante ouviu a explicação dos conceitos área e perímetro de quadrados e descrição das etapas da construção do fractal Tapete de Sierpinski. A atividade realizada pela turma envolveu pintura com lápis de cor e a estudante com baixa visão usou o material recortado e colado sobre bases descrito na seção anterior.

A ação pedagógica foi dividida em três momentos, que são descritos a seguir. No primeiro momento, foi investigado se a estudante sabia utilizar a régua adaptada, entregando uma para ela manusear. Mostrando que a régua está dividida em pequenas 
partes, foi feita a seguinte pergunta: Quanto mede cada uma das pequenas partes? A estudante não lembrava como utilizar a régua e por isso foi solicitado que ela sentisse as marcações em alto relevo e explicado que cada uma das pequenas partes mede um centímetro.

Em seguida, foram entregues alguns recortes com a forma de diferentes figuras geométricas sendo solicitado que a estudante escolhesse entre as figuras aquelas que têm a forma de um quadrado. Nesta atividade a estudante teve facilidade em identificar as formas geométricas, logo retirando da mesa o quadrado, sem manusear as outras figuras, porque foram recortadas com tamanho aproximado de uma mão, bem coloridas e dispostas sobre fundo branco. Quando indagada sobre os critérios que usou para retirar da mesa o quadrado, ela explicou com suas palavras e com gestos que ele tem os lados com mesma medida. Em seguida, foi solicitado que ela calculasse a medida dos lados do quadrado utilizando a régua entregue, sendo auxiliada na medição. Após ter a medida do lado do quadrado, correspondente a $17 \mathrm{~cm}$, a estudante calculou o perímetro, mas encontrou dificuldade na adição. Ela comentou que compreendeu o que foi solicitado e afirmou que não conseguia realizar o cálculo. Indagada se realiza os cálculos com sorobã ou calculadora adaptada, ela respondeu que não usava estes instrumentos. Tendo baixa visão, realiza cálculos sobre papel, escrevendo com pincel marcador permanente símbolos matemáticos bem grandes. Através destas atividades verificamos que a estudante apresentou dificuldades em utilizar a régua adaptada, precisando do nosso auxílio para manuseá-la corretamente e, em relação à identificação de quadrados entre várias figuras geométricas, ela não apresentou dificuldades.

Num segundo momento, foi iniciada a atividade envolvendo a primeira iteração do fractal Tapete de Sierpinki, sendo entregue a primeira maquete do material didático para exploração tátil. Verbalmente, a estudante descreveu que tinha em mãos um quadrado dividido em pequenos quadradinhos. Foi enfatizado que cada um dos quadrados pequenos mede $1 \mathrm{~cm}$ de lado e solicitado que ela contasse quantos quadrados pequenos havia em um fileira do quadrado grande. Ela realizou a contagem dos quadradinhos e informou que há 18 em uma fileira. Sendo observado que a medida do lado de cada quadrado pequeno é $1 \mathrm{~cm}$, o quadrado maior tem $18 \mathrm{~cm}$, e, nesse momento, a estudante mediu com a régua adaptada o lado do quadrado maior, conferindo que a medida é $18 \mathrm{~cm}$ de lado. Em seguida, 
foi solicitado o cálculo do perímetro desse quadrado maior, mas foi necessário explicar o significado da palavra "perímetro", relembrando que se trata da soma dos lados. A estudante expressou verbalmente que compreendeu o conceito perímetro de um quadrado, mas afirmou que não conseguia realizar a soma 18+18+18+18.

Posteriormente, foi entregue um quadrado de $1 \mathrm{~cm}$ de lado para que a estudante sentisse como sua área é pequena, sendo explicado que a área desse quadrado pequeno é $1 \mathrm{~cm}^{2}$. A estudante pode perceber que a área do quadrado maior é a quantidade de quadrados pequenos que cabem dentro desta região plana. Foi solicitado que ela descrevesse como faria para conhecer a área do quadrado maior e ela respondeu que podem ser contados quantos quadrados pequenos cabem no quadrado grande. Foi explicado que a multiplicação da medida de um lado pela medida de outro lado também fornece a área do quadrado grande, mas não tendo pincel marcador e papel disponíveis, o cálculo não foi realizado.

Em seguida, foi entregue para a estudante a segunda maquete do material didático, sendo relembrado que, para construir a primeira iteração do fractal Tapete de Sierpinski, o quadrado deve ser dividido em nove partes com o mesmo tamanho e deve ser retirado 0 quadrado central. A estudante tateou as marcações percebendo os nove quadrados marcados e, para o entendimento da primeira iteração, foi deixado o quadrado central sem colar, apenas encaixado sobre a base, para que ela pudesse removê-lo. Após, foi solicitado que medisse o lado do quadrado menor, chegando à medida de $6 \mathrm{~cm}$ de lado. Novamente foi auxiliada no posicionamento da régua, sendo repetido o equívoco de iniciar a medida não no zero, mas no início do objeto. Sendo solicitado o cálculo da área deste quadrado, foi discutido novamente que a área pode ser calculada multiplicando um lado pelo outro; assim, como o lado do quadrado pequeno mede $6 \mathrm{~cm}$, e como todos os lados medem $6 \mathrm{~cm}$, para saber a área foi efetuada a multiplicação 6 × 6 . O cálculo foi realizado de modo correto mostrando que domina a tabuada de 6.

Após, foi pedido que ela descrevesse quantos quadrados existem na primeira iteração desse fractal e ela respondeu que há 8 peças quadradas. Foi discutido que para calcular a área total do fractal Tapete de Sierpinski na primeira iteração que estava sendo realizada, como sabe-se que a área de cada um destes quadrados é $36 \mathrm{~cm}^{2}$, a área total pode ser calculada somando-se 36 oito vezes, ou multiplicando-se 36 por 8. 
Neste momento foi entregue a terceira maquete do material didático, com a representação da segunda iteração do fractal Tapete de Sierpinski. A estudante tateou e, ao ser indagada sobre a diferença desse material em relação ao anterior, ela respondeu que tem quadrados menores, mas o quadrado do meio é do mesmo tamanho que o anterior. Foi relembrado que, para construir a iteração dois do Tapete de Sierpinski, cada quadrado é dividido em nove partes com o mesmo tamanho e é retirado cada quadrado central de cada pequeno quadrado. Em seguida, foi pedido para ela medir o lado do quadrado pequeno e calcular a área do mesmo. Para medir o lado, ela apresentou a mesma dificuldade anteriormente detectada, entendendo que a medida deve ser iniciada não no zero, mas no início da régua. Foi auxiliada no posicionamento da régua e realizou a medida de modo correto. Conversando sobre o cálculo da área total do fractal Tapete de Sierpinski na segunda iteração, a estudante explicou que pode ser obtida por meio da contagem, um a um, de todos os pequenos quadrados. No entanto, não comentou que poderia ser efetuada uma multiplicação ou a soma das áreas parciais.

No último momento, após a pausa para descanso e merenda, foi aplicada uma atividade que teve como objetivo verificar se a estudante compreendeu como são realizadas as primeiras iterações do fractal Tapete de Sierpinski. Primeiramente foi entregue uma maquete constituída por uma base sobre a qual foi colada uma moldura tendo como interior o formato de um quadrado, medindo $24 \mathrm{~cm}$ de lado internamente, e nove quadrados recortados em E.V.A, medindo $8 \mathrm{~cm}$ de lado. A estudante manipulou as peças quadradas, a base e tateou a moldura. Foi pedido para ela representar a primeira iteração do fractal Tapete de Sierpinski, que foi estudada com a utilização da segunda maquete. No início, ela não conseguiu montar, então entregamos novamente a maquete com a primeira iteração que foi utilizada anteriormente para relembrar a construção e, em seguida, ela conseguiu realizar a iteração encaixando os quadrados de modo correto. Para finalizar a atividade, pedimos para ela medir o lado do quadrado pequeno e calcular sua área. A régua foi novamente posicionada no início e não no zero e a estudante encontrou dificuldade na realização do cálculo que envolvia a tabuada de 8 . A estudante comentou que domina bem a tabuada até 7 .

A manipulação do material possibilitou o entendimento dos conceitos de área e perímetro de quadrados e facilitou a construção do fractal Tapete de Sierpinski. Para a 
realização dos cálculos, a estudante optava pela contagem, um a um, dos pequenos quadrados que compõem as diversas iterações. Assim, a presença de um grande número de pequenos quadrados tornava o processo de contagem extremamente demorado, mostrando a importância da memorização de todas as tabuadas.

\section{Considerações Finais}

Todos somos diferentes e apresentamos dificuldades educacionais, como também temos habilidades e possibilidades diferenciadas. Por isso, um ensino tradicional que não considera as diferenças e as necessidades de todos, não facilita a aprendizagem. A utilização de materiais diferenciados pode contribuir no ensino e aprendizagem dos estudantes, principalmente dos que têm alguma deficiência.

Conforme foi apresentado neste artigo, para a aprendizagem do estudante com deficiência visual, é necessário possibilitar o uso do sentidos remanescentes e do diálogo correto, evitando a comunicação visual e gestual. No caso do material descrito neste artigo, o E.V.A. apresenta a grande vantagem de poder ser marcado com linhas em baixo relevo, que facilmente são tateadas, possibilitando o entendimento da construção do fractal Tapete de Sierpinski. De acordo com estas informações e as orientações de Sá, Campos e Silva (2007), para elaborações de materiais didáticos a pessoas com deficiência visual, o material didático utilizado nesta atividade foi confeccionado com materiais que não oferecem perigo aos estudantes e são de fácil manuseio, como também podem ser utilizados por todos os estudantes, não apenas pelos com deficiência visual. O presente estudo de caso envolveu uma estudante com baixa visão e a escolha de cores fortes e recorte de figuras de tamanho adequado às dimensões da mesa escolar.

O material confeccionado trouxe os resultados desejados, possibilitando 0 entendimento dos conceitos de área e de perímetro de quadrados. Especial atenção deve ser dada ao correto posicionamento da régua adaptada, sendo necessário verificar se o ponto onde está representado o número zero está coincidindo com um vértice do quadrado cujo lado está sendo medido.

No caso desse material ser usado com o objetivo de realização de cálculos envolvendo adições e multiplicações, deve ser usado um instrumento, por exemplo, sorobã 
DOI: $10.5902 / 1984686 \times 26287$

ou calculadora adaptada, ou, no caso de estudantes com baixa visão, talvez possam ser usados uma lupa ou pincel marcador permanente de cor escura sobre papel branco.

\section{Referências}

BARBOSA, R. M. Descobrindo a Geometria Fractal: para a sala de aula. 3. ed. Belo Horizonte: Autêntica, 2005.

BICUDO, M. A. V. A filosofia da Educação centrada no aluno. In: MARTINS, J. BICUDO, M. A.V. Estudos sobre existencialismo, fenomenologia e educação.2 ed. São Paulo: Centauro, 2006. p. 57-101.

BRASIL. Portaria no 3.128, de 24 de dezembro de 2008. Define Que As Redes Estaduais de Atenção à Pessoa Com Deficiência Visual Sejam Compostas Por Ações na Atenção Básica e Serviços de Reabilitação Visual. Brasília, 2008. Disponível em: <http://bvsms.saude.gov.br/bvs/saudelegis/gm/2008/prt3128_24_12_2008.html>. Acesso em: 04 nov. 2016.

Subsecretaria Nacional de Promoção dos Direitos da Pessoa com Deficiência. Comitê de Ajudas Técnicas. Tecnologia Assistiva. Brasília: CORDE, 2009. Disponível em: <http://www.pessoacomdeficiencia.gov.br/app/sites/default/files/publicacoes/livrotecnologia-assistiva.pdf>. Acesso em: 23 nov. 2017.

CENTRO DE FÍSICA TEÓRICA E COMPUTACIONAL. O tapete de Sierpinski.

Disponível

em: <http://cftc.cii.fc.ul.pt/PRISMA/capitulos/capitulo2/modulo4/topico5.php>.Acesso em: 17 out. 2016.

DANTE, L. R. Tudo é matemática: 6ª série. São Paulo: Ática, 2002a. (Coleção Tudo é Matemática, 2).

Tudo é matemática: $7^{a}$ série. São Paulo: Ática, 2002b.(Coleção Tudo é Matemática, 3).

Matemática: livro do professor. São Paulo: Ática, 2004.

KARAS, E. W. (Org.). Matemática: fractais: propriedades e construção. Curitiba: PPGE/UFPR, $2008 . \quad$ Disponível em: <http://www.matematica.seed.pr.gov.br/arquivos/File/manuais/livreto_matematica_uf pr_fractais.pdf>. Acesso em: 11 out. 2016.

MANDELBROT, B. Objectos Fractais. 2. ed. Lisboa: Gradiva, 1998.

SÁ, E. D.; CAMPOS, I. M.; SILVA, M. B. C. Atendimento educacional especializado: deficiência visual. SEESP / SEED / MEC Brasília, 2007. Disponível em: <http://portal.mec.gov.br/seesp/arquivos/pdf/aee_dv.pdf>. Acesso em: 10 set. 2016. 
SHIMAZAKI, E. M.; SILVA, S. C. R.; VIGINHESKI, L. V. M. O ensino de Matemática e a diversidade: o caso de uma estudante com deficiência visual. Interfaces da Educação, Paranaíba, v. 6, n. 18, p.148-164, set./dez. 2016. Disponível em: $<$ http://periodicosonline.uems.br/index.php/interfaces/article/view/1082/913>. Acesso em: 21 nov. 2016.

\section{Correspondência}

Ana Paula Poffo Koepsel - Fundação Universidade Regional de Blumenau (FURB). R. Antônio da Veiga, 140 - Victor Konder, CEP: 89030-903, Blumenau, Santa Catarina, Brasil.

\section{(C) $(1) \Theta$}

Th is work is licensed under a Creative Commons Attribution-NonCommercial 4.0 International (CC BY-NC 4.0) 